\title{
Agreement between inferior vena cava diameter measurements by subxiphoid versus transhepatic views
}

\author{
Atul Prabhakar Kulkarni, S. Janarthanan, Harish M. M, Siddique Suhail, Harish Chaudhari, \\ Vandana Agarwal' ${ }^{1}$, Vijaya P. Patil ${ }^{1}$, Jigeeshu V. Divatia ${ }^{1}$
}

Context: Correcting hypovolemia is extremely important. Central venous pressure
measurement is often done to assess volume status. Measurement of inferior vena cava
(IVC) is conventionally done in the subcostal view using ultrasonography. It may not be
possible to obtain this view in all patients. Aims: We therefore evaluated the limits of
agreement between the IVC diameter measurement and variation in subcostal and that by
the lateral transhepatic view. Settings and Design: Prospective study in a tertiary care
referral hospital intensive care unit. Subjects and Methods: After Institutional Ethics
Committee approval and informed consent, we obtained I 75 paired measurements of
the IVC diameter and variation in both the views in adult mechanically ventilated patients.
The measurements were carried out by experienced researchers. We then obtained the
limits of agreement for minimum, maximum diameter, percentage variation of IVC in
relation to respiration. Statistical Analysis Used: Bland-Altman's limits of agreement
to get precision and bias. Results: The limits of agreement were wide for minimum and
maximum IVC diameter with variation of as much as 4 mm in both directions. However,
the limits of agreement were much narrower when the percentage variation in relation to
respiration was plotted on the Bland-Altman plot. Conclusions: We conclude that when
it is not possible to obtain the subcostal view, it is possible to use the lateral transhepatic
view. However, using the percentage variation in IVC size is likely to be more reliable than
the absolute diameter alone. It is possible to use both views interchangeably.
Keywords: Fluid responsiveness, inferior vena cava collapsibility, inferior vena cava
diameter, inferior vena cava distensibility, limits of agreement

\section{Introduction}

Hypovolemia is frequent in critically ill patients and may result in inadequate tissue perfusion and subsequent multi-organ failure. The estimation of intravascular volume status as well as fluid responsiveness is hence an important issue. Fluid replacement is an important intervention in these patients; however, excessive fluid

From:

Division of Critical Care Medicine, Department of Anaesthesia Critical Care and Pain, Tata Memorial Hospital, ${ }^{1}$ Department of Anaesthesia Critical Care and Pain, Tata Memorial Hospital, Mumbai, Maharashtra, India

\section{Correspondence:}

Dr. Atul Prabhakar Kulkarni, Division of Critical Care Medicine,

Department of Anaesthesiology Critical Care and Pain, Tata Memorial Hospital,

Dr. E. Borges Road, Parel, Mumbai - 400 012, Maharashtra, India.

E-mail: kaivalyaak@yahoo.co.in

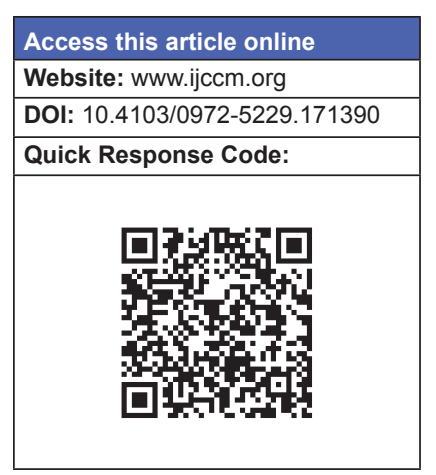

infusion and an increase in net positive fluid balance lead to need for prolonged mechanical ventilation and increased mortality. ${ }^{[1,2]}$ Therefore, it is essential to provide adequate fluid resuscitation without "overloading" in order to improve the outcome of the critically ill patients.

This is an open access article distributed under the terms of the Creative Commons Attribution-NonCommercial-ShareAlike 3.0 License, which allows others to remix, tweak, and build upon the work non-commercially, as long as the author is credited and the new creations are licensed under the identical terms.

For reprints contact: reprints@ @medknow.com

How to cite this article: Kulkarni AP, Janarthanan S, Harish MM, Suhail S, Chaudhari H, Agarwal V, Patil VP, Divatia JV. Agreement between inferior vena cava diameter measurements by subxiphoid versus transhepatic views. Indian J Crit Care Med 2015;19:719-22. 
This requires a careful assessment of the intravascular volume status and fluid responsiveness prior to the institution of fluid therapy. A variety of static and dynamic parameters have been used to assess fluid responsiveness. Over the last few years, bedside ultrasound (BUS) is being increasingly used by the intensivists to assess the intravascular volume status and fluid responsiveness by measuring inferior vena cava (IVC) diameter and variation in IVC diameter in relation to respiration. ${ }^{[3]}$ In spontaneously breathing patients, the IVC diameter reduces during inspiration, whereas with positive pressure ventilation, the IVC diameter increases during inspiration, enabling calculation of indices such as dIVC, collapsibility, and distensibility index. ${ }^{[4]}$ The actual IVC diameter measured on M-mode is correlated with the right atrial pressure. However, the variation in the size of the IVC, i.e. dIVC, collapsibility, and distensibility index, can be used to assess fluid responsiveness. The conventional view used to measure IVC diameter and variation in size is the subxiphoid view. Obtaining this view may be difficult in obese patients and those who have undergone abdominal surgery. We wanted to see if the transhepatic view of IVC can be an alternative to the subxiphoid view. The result of the study will add one more echocardiographic view to our armamentarium, and we will still be able to assess intravascular volume status even when the traditional subxiphoid view is not obtainable. We therefore evaluated the agreement between the measurement of IVC diameters and collapsibility/distensibility indices by the subcostal view and a lateral transhepatic view.

\section{Subjects and Methods}

This single center study was conducted prospectively in mechanically ventilated adult critically ill patients at a tertiary referral center. Hospital Ethics Committee approval was obtained for either deferred consent or for a consent from a surrogate. After informed consent, we included 88 patients in shock of any origin and in whom need for fluid infusion was anticipated and both subcostal and transhepatic views were obtainable. We excluded patients in whom either of the views was not possible for any reason or who did not give informed consent. For obtaining the subcostal view, patients were placed in supine position and the four-chamber view was obtained using a cardiac probe of the Sonosite ${ }^{\mathrm{TM}}$ (M-Turbo model) ultrasonography (USG) machine. Then by rotating and swinging the BUS probe to the right, the IVC was visualized and the diameter measured at the point of entry of the hepatic vein into the IVC. For the lateral transhepatic view, we used liver as an acoustic window, by placing the probe in the right anterior mid-axillary line, similar to the placement for evaluating Morison's pouch, in the Focused Assessment with Sonography for Trauma examination. The marker on probe pointed toward the head of the patient. By scanning more anteriorly and cephalad than the Morison's pouch view, the IVC was visualized running longitudinally adjacent to the liver and crossing the diaphragm. Again, the measurement of diameter was done at the entry of hepatic vein in IVC. The IVC collapsibility index during spontaneous breathing was expressed as the difference between the value of the maximum diameter and the minimum diameter, divided by the maximum of the two values. dIVC or $\triangle \mathrm{IVC}$ was quantified by measuring the difference between the maximum and minimum diameters on the M-mode tracing and dividing it by the mean of the two. The denominator here is the mean diameter. The IVC distensibility index was measured during controlled mechanical ventilation and expressed as the difference between the value of the maximum diameter and the minimum diameter, divided by the minimum of the two values. ${ }^{[5-7]}$ We obtained 175 paired measurements, two sets in one patient being obtained by two researchers at the same time. The researchers had been trained at a 2 days workshop on transthoracic echocardiography and subsequently had more than 2 years' experience in BUS. In these 2 years, they had performed more than 100 bedside transthoracic echocardiography procedures each. They were blinded to the measurements made by each other, and later we took mean of both readings.

\section{Statistics}

Bland-Altman analysis was performed to determine the precision, bias, and limits of agreement between two methods of IVC diameter measurement. These were determined for the minimum and maximum IVC diameter as well as the variability in IVC diameter with respiration.

\section{Results}

In this prospective study, a total of 175 paired readings were obtained by two researchers blinded to each other's findings. The average of the values obtained by both observers was calculated. Limits of agreement were assessed separately for maximum diameter, minimum diameter, and variability. The limits of agreement were wide when both the minimum diameter and maximum diameter were plotted on the Bland-Altman graph [Figures 1 and 2]. For minimum diameter measured, the bias was 0.17 and the limits of agreement were 0.4 and -0.37 . For maximum diameter, the bias was 0.027 and the limits of agreement were 0.4 and -0.36 . For the percentage variability of the IVC diameter, the bias was 0.005 and the limits of agreement were 0.17 and -0.16 [Figure 3]. These limits of agreement were much narrower when compared with the maximum and minimum values of the IVC diameter. 


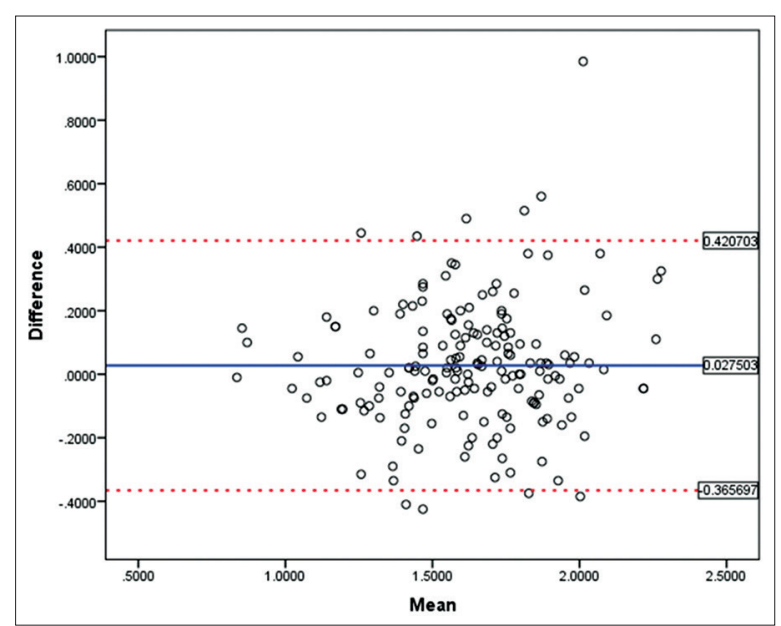

Figure I: Limits of agreement for average minimum IVC diameter

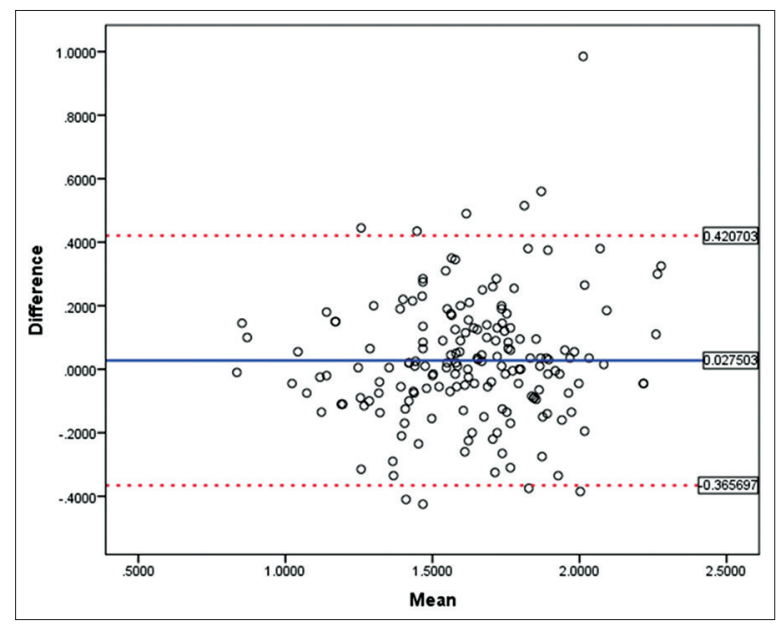

Figure 2: Limits of agreement for average maximum IVC diameter

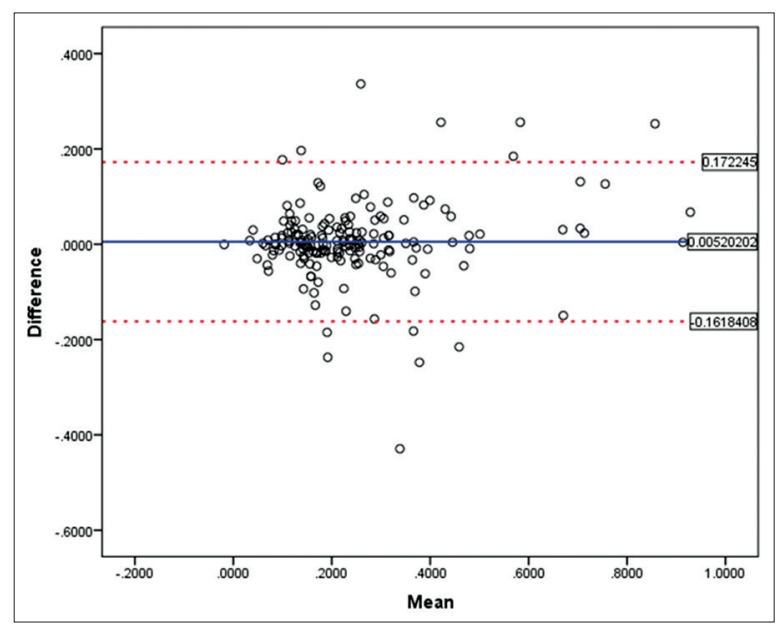

Figure 3: Limits of agreement for average for variability in IVC diameter

\section{Discussion}

The minimum and maximum IVC diameter measured at two different sites differed by nearly by $4 \mathrm{~mm}$ on either side of the bias. The limits of agreement were narrower when the variation in size with respiration was taken into account. Hence, even if actual diameter value is not very useful, a change in diameter with respiration can be substituted when it is not possible to obtain either view. In the past years, many clinical and experimental studies led to the validation in mechanically ventilated patients of a few "dynamic" parameters based on heart-lung interactions. Assessing IVC diameter has been one of the methods to identify patients who are fluid responsive. A significant dilation of the IVC during tidal ventilation accurately predicts fluid responsiveness. ${ }^{[5,6]}$ The IVC is a highly collapsible major vein, and its diameter closely correlates with right side cardiac function. ${ }^{[8]}$ The IVC diameter has not been found to be affected by body's compensatory vasoconstrictor response to volume loss. ${ }^{[9]}$ Hence, it reflects volume status more closely than other hemodynamic parameters such as blood pressure, pulse rate, diameter of aorta, and others. Studies in the past have shown the usefulness of IVC diameter in monitoring volume status in patients undergoing hemodialysis and in patients under mechanical ventilation in Intensive Care Units (ICUs). ${ }^{[10]}$ To determine the status of intravascular volume, the dIVC measurement is one of the methods. ${ }^{[11]}$ Yanagawa et al. used the dIVC expiratory (dIVCe) in the early diagnosis of hypovolemic shock using USG in patients with trauma. ${ }^{[12]}$ In this study, the threshold level of the dIVCe for the diagnosis of hemorrhagic shock was $9 \mathrm{~mm}$. The dIVCe $(7.7 \pm 0.3 \mathrm{~mm})$ measured in the shock group was significantly lower than the level $(13.4 \pm 0.7 \mathrm{~mm})$ measured in the control group. A similar study was performed by Sefidbakht et al. in patients with trauma. ${ }^{[13]}$ In this study, dIVCe and dIVCi levels $(5.6 \pm 0.8$ and $4 \pm 0.7 \mathrm{~mm}$ ) of the shock group were also significantly lower than those of the control group (11.9 \pm 2.2 and $9.6 \pm 2 \mathrm{~mm})$, and the caval index (CI) was higher. These studies suggest the dIVC can be considered a reliable indicator of shock, although blood pressure is within normal limits because of sympathetic activation. Akilli et al. compared the dIVC with other shock parameters such as heart rate, systolic blood pressure/diastolic blood pressure, shock index, urine output, hemoglobin level, leukocyte count, and lactate and base excess in the patients admitted to the emergency department with hemorrhagic shock. They concluded that the dIVC in hemorrhagic shock was more valuable than in conventional shock parameters. ${ }^{[14]}$ Schefold et al. studied IVC diameter correlation with invasive hemodynamic measures in mechanically ventilated ICU patients with sepsis. ${ }^{[15]}$ IVC diameters were found to correlate with central venous pressure (CVP), extravascular lung water index, intrathoracic blood volume index, the intrathoracic thermal volume, and the $\mathrm{PaO}_{2} / \mathrm{FiO}_{2}$ oxygenation index. Therefore, sonographic determination of IVC diameter seems useful in the early assessment of fluid status in 
mechanically ventilated septic patients. Though IVC diameter variability can be used for fluid responsiveness, there are difficulties in assessing it in obese patients, in patients who had undergone abdominal surgeries and in whom IVC is difficult to visualize by standard subcostal view. The transhepatic view provides an alternative for this by assessing the IVC using liver as the acoustic window. We found wide limits of agreement when we plotted the minimum and max diameter. This difference could be due to the interrater variability and difference in angle of viewing. Fields et al. reported that the intraclass correlation coefficient of the IVC was 0.81 (95\% CI, 0.67-0.89) for maximum IVC diameter and intraclass correlation coefficient was 0.77 (95\% CI, 0.62-0.87) for minimum IVC diameter. ${ }^{[16]}$ They also stated that the interrater reliability of measurement was increased from 0.49 to 0.81 after expiration and from 0.51 to 0.85 after inspiration at the end of the first 5 examinations. They study suggested that 15 USA examinations may be necessary to obtain sufficient skill. Akkaya et al. found that there was an increase in the interrater reliability of IVC-min measurements after 10 examinations. ${ }^{[17]}$ In our study, the researchers who measured the IVC diameters had at least 2 years of experience in BUS. Hence, the difference in diameter between the two views may have been because of a change in angle of looking at the IVC. It is also logical that the percentage variation had narrower limits of agreement since we were not looking at a number but a qualitative percentage change in the diameter.

Other factors can affect IVC diameter and its relationship to CVP. The liver or diaphragm may tend to tether the IVC in the "open" position in the most proximal portions but there is variation in the diameter of the IVC among normal patients, so it seems the tethering must not be preventing the change in IVC size. Changes in intraabdominal and intrathoracic pressures (e.g., during positive-pressure ventilation or Valsalva maneuver) are also well known to alter the CVP and have some effect on IVC diameters. Whether any of these factors does affect our ability at the bedside to infer CVP from the IVC diameter has not been well studied. We need to keep these limitations in mind.

Therefore, we believe when the subcostal view is difficult to obtain, we can use the lateral transhepatic view, but instead of using actual diameter, we should use variation in diameter to determine volume status of the patient.

\section{Conclusions}

We conclude that when it is not possible to obtain the subcostal view, it is possible to use the lateral transhepatic view. However, using the percentage variation in IVC size is likely to be more reliable than the absolute diameter alone. It is possible to use both views interchangeably.

\section{Financial support and sponsorship}

Nil.

\section{Conflicts of interest}

There are no conflicts of interest.

\section{References}

1. Boyd JH, Forbes J, Nakada TA, Walley KR, Russell JA. Fluid resuscitation in septic shock: A positive fluid balance and elevated central venous pressure are associated with increased mortality. Crit Care Med 2011;39:259-65.

2. Vincent JL, Sakr Y, Sprung CL, Ranieri VM, Reinhart K, Gerlach H, et al. Sepsis in European intensive care units: Results of the SOAP study. Crit Care Med 2006;34:344-53.

3. Kitakule MM, Mayo P. Use of ultrasound to assess fluid responsiveness in the intensive care unit. Open Crit Care Med J 2010;3:33-7.

4. American College of Emergency Physicians. American College of Emergency Physicians. ACEP emergency ultrasound guidelines-2001. Ann Emerg Med 2001;38:470-81.

5. Barbier C, Loubières Y, Schmit C, Hayon J, Ricôme JL, Jardin F, et $a l$. Respiratory changes in inferior vena cava diameter are helpful in predicting fluid responsiveness in ventilated septic patients. Intensive Care Med 2004;30:1740-6.

6. Feissel M, Michard F, Faller JP, Teboul JL. The respiratory variation in inferior vena cava diameter as a guide to fluid therapy. Intensive Care Med 2004;30:1834-7.

7. Zengin S, Al B, Genc S, Yildirim C, Ercan S, Dogan M, et al. Role of inferior vena cava and right ventricular diameter in assessment of volume status: A comparative study: Ultrasound and hypovolemia. Am J Emerg Med 2013;31:763-7.

8. Moreno FL, Hagan AD, Holmen JR, Pryor TA, Strickland RD, Castle CH. Evaluation of size and dynamics of the inferior vena cava as an index of right-sided cardiac function. Am J Cardiol $1984 ; 53: 579-85$.

9. Nette RW, Ie EH, Vletter WB, Krams R, Weimar W, Zietse R. Norepinephrine-induced vasoconstriction results in decreased blood volume in dialysis patients. Nephrol Dial Transplant 2006;21:1305-11.

10. Mandelbaum A, Ritz E. Vena cava diameter measurement for estimation of dry weight in haemodialysis patients. Nephrol Dial Transplant 1996;11 Suppl 2:24-7.

11. Dipti A, Soucy Z, Surana A, Chandra S. Role of inferior vena cava diameter in assessment of volume status: A meta-analysis. Am J Emerg Med 2012;30:1414-1419.e1.

12. Yanagawa Y, Nishi K, Sakamoto T, Okada Y. Early diagnosis of hypovolemic shock by sonographic measurement of inferior vena cava in trauma patients. J Trauma 2005;58:825-9.

13. Sefidbakht S, Assadsangabi R, Abbasi HR, Nabavizadeh A. Sonographic measurement of the inferior vena cava as a predictor of shock in trauma patients. Emerg Radiol 2007;14:181-5.

14. Akilli B, Bayir A, Kara F, Ak A, Cander B. Inferior vena cava diameter as a marker of early hemorrhagic shock: A comparative study. Ulus Travma Acil Cerrahi Derg 2010;16:113-8.

15. Schefold JC, Storm C, Bercker S, Pschowski R, Oppert M, Krüger A, et al. Inferior vena cava diameter correlates with invasive hemodynamic measures in mechanically ventilated intensive care unit patients with sepsis. J Emerg Med 2010;38:632-7.

16. Fields JM, Lee PA, Jenq KY, Mark DG, Panebianco NL, Dean AJ. The interrater reliability of inferior vena cava ultrasound by bedside clinician sonographers in emergency department patients. Acad Emerg Med 2011;18:98-101.

17. Akkaya A, Yesilaras M, Aksay E, Sever M, Atilla OD. The interrater reliability of ultrasound imaging of the inferior vena cava performed by emergency residents. Am J Emerg Med 2013;31:1509-11. 